\title{
BMJ Open REducing unwarranted variation in the Delivery of high qUality hip fraCture services in England and Wales (REDUCE): protocol for a mixed- methods study
}

\author{
Rita Patel (10 , ', Sarah Drew (D) , ${ }^{1}$ Antony Johansen, ${ }^{2,3}$ Tim Chesser (D) , ${ }^{4}$ \\ Muhammad K Javaid (D) , ${ }^{5}$ Xavier L Griffin (D) , 6,7 Tim Jones, ${ }^{8}$ Jill Griffin, ${ }^{8}$ \\ Marianne Bradshaw, ${ }^{1}$ Katie Whale (1) , ${ }^{1,9}$ Estela Capelas Barbosa (D) , ${ }^{1}$ \\ Elsa M R Marques (D) , ${ }^{1,9}$ Yoav Ben-Shlomo (D) , ${ }^{10}$ Rachael Gooberman-Hill (D) , ,,9 \\ Andrew Judge (i) , ${ }^{1,5,9}$ Celia L Gregson (1) ${ }^{1}$
}

To cite: Patel $R$, Drew $S$ Johansen $A$, et al. REducing unwarranted variation in the Delivery of high quality hip fraCture services in England and Wales (REDUCE): protocol for a mixedmethods study. BMJ Open 2021;11:e049763. doi:10.1136/ bmjopen-2021-049763

- Prepublication history for this paper is available online. To view these files, please visit the journal online (http://dx.doi. org/10.1136/bmjopen-2021049763).

Received 02 February 2021 Revised 19 April 2021 Accepted 20 April 2021

Check for updates

(C) Author(s) (or their employer(s)) 2021. Re-use permitted under CC BY. Published by BMJ.

For numbered affiliations see end of article.

Correspondence to

Dr Celia L Gregson;

celia.gregson@bristol.ac.uk

\section{ABSTRACT}

Introduction Substantial variation in the delivery of hip fracture care, and patient outcomes persists between hospitals, despite established UK national standards and guidelines. Patients' outcomes are partly explained by patient-level risk factors, but it is hypothesised that organisational-level factors account for the persistence of unwarranted variation in outcomes. The mixed-methods REducing unwarranted variation in the Delivery of high qUality hip fraCture services in England and Wales (REDUCE) study, aims to determine key organisational factors to target to improve patient care.

Methods and analysis Quantitative analysis will assess the outcomes of patients treated at 172 hospitals in England and Wales (2016-2019) using National Hip Fracture Database data combined with English Hospital Episodes Statistics; Patient Episode Database for Wales; Civil Registration (deaths) and multiple organisationallevel audits to characterise each service provider. Statistical analyses will identify which organisational factors explain variation in patient outcomes, and typify care pathways with high-quality consistent patient outcomes. Documentary analysis of 20 anonymised British Orthopaedic Association hospital-initiated peerreview reports, and qualitative interviews with staff from four diverse UK hospitals providing hip fracture care, will identify barriers and facilitators to care delivery. The COVID-19 pandemic has posed a major challenge to the resilience of services and interviews will explore strategies used to adapt and innovate. This system-wide understanding will inform the development, in partnership with key national stakeholders, of an 'Implementation Toolkit' to inform and improve commissioning and delivery of hip fracture services.

Ethics and dissemination This study was approved: quantitative study by London, City and East Research Ethics Committee (20/L0/0101); and qualitative study by Faculty of Health Sciences University of Bristol Research Ethics Committee (Ref: 108284), National Health Service (NHS) Health Research Authority (20/HRA/71) and
Strengths and limitations of this study

- A mixed-methodology approach will aid identification of hospital-level organisational factors which explain adverse patient outcomes following hip fracture, and which are amenable to improvement across the UK.

- This study is novel in terms of its scale and the unique datasets used, which gives a rare opportunity to robustly assess a complex system of care and the impacts this system has on patients with hip fracture and National Health Service (NHS) budgets.

- Use of quantitative, economic and qualitative analysis will provide a system-wide understanding of the hip fracture care pathway, which will inform development of an Implementation Toolkit, in partnership with key national stakeholders, to improve future service design.

- While multiple organisational datasets exist relevant to patient care in NHS hospitals, linking this NHS activity to comprehensive social care data is not viable, hence, social care sequalae following hip fracture admission does not form part of this protocol.

- Currently the NHS is experiencing unprecedented pressures; the REducing unwarranted variation in the Delivery of high qUality hip fraCture services in England and Wales study will determine the most efficient management pathways for high cost patients, to improve patient outcomes and free NHS resources for use elsewhere.

each NHS Trust provided Research and Development approval. Findings will be disseminated through scientific conferences, peer-reviewed journals and online workshops.

\section{INTRODUCTION}

Each year in the UK more than 70000 older adults sustain a fragility fracture of the hip. ${ }^{1}$ 
Such fractures are indicative of osteoporosis. ${ }^{1}$ Hip fractures are costly to patients, relatives and the National Health Service (NHS), with a significant impact on quality of life ${ }^{2}$; a quarter of patients die within 1 year of hip fracture. ${ }^{3}$ Research has shown annual NHS medical costs from hip fracture exceed $£ 1.2$ billion. ${ }^{4}$

Patients sustaining hip fractures almost invariably require an operation, but patient care has many complexities requiring contributions from various healthcare professionals at different time-points during an oftenlengthy treatment journey. Many guidelines have been published trying to ensure all components of the care of patients sustaining hip fractures (hip fracture care) are provided consistently and to a high standard in all hospitals. ${ }^{15-7}$ While care has improved for some, there remains a great deal of variation across the UK in how health services deliver hip fracture care, so treatment still depends on where and when patients present to hospital. This unwarranted variation in care includes delays waiting for an operation, the type of operation performed, how much specialist help is provided, how soon physiotherapy is delivered, how thoroughly bone health is assessed, and more.

Substantial variation exists in how well patients recover after a hip fracture. Across the 172 hospitals currently providing hip fracture care in England and Wales, 1 month after hip fracture the proportion of patients who have died varies from $2 \%$ to $14 \%$ between hospitals, ${ }^{8}$ and the proportion who have been able to return home ranges from $29 \%$ to $85 \% .{ }^{9}$ While overall $61 \%$ of patients are prescribed medication to reduce the chance of a further fracture in the future, this can vary enormously from $6 \%$ to $99.5 \%$ according to the hospital delivering hip fracture care. ${ }^{10}$ Time spent in hospital is highly variable (the median length of stay in acute and postacute NHS care is 17 days, IQR 10-30 days) and the chance of being readmitted to hospital within 30 days because of a deterioration after discharge is high at $16 \% .{ }^{11}{ }^{12}$ While patient outcomes are partly explained by patient-level risk factors (eg, age and comorbidity), it is hypothesised that organisational factors are responsible for variation in the delivery of fracture care pathways and hence patient outcomes; these organisational factors are potentially modifiable.

It is important to understand how the set-up and organisation of healthcare services affects patient recovery and outcomes after hip fracture. These services can vary in many ways, for example, types and grade of clinical staff; capacity to perform prompt operations; access to suitable rehabilitation services. It is expected these factors will explain variation in quality of care, patient outcomes and associated health costs. Understanding these will enable us to inform changes in healthcare services to minimise avoidable variation in fracture care and improve the quality of care for all patients across the country.

The 2020 COVID-19 pandemic has had an unprecedented impact on health service delivery across the NHS. ${ }^{13}{ }^{14}$ Hospitals have needed to rapidly adapt and reorganise services to continue to deliver hip fracture care. To assist in service planning, NHS England ${ }^{1516}$ and the British Orthopaedic Association (BOA) ${ }^{17}$ issued rapid guidance in March 2020 on the management of hip fracture patients during the coronavirus pandemic. ${ }^{15-17}$ The limited evidence to date suggests that there has been wide variation in how hospitals have reconfigured services ${ }^{18} 19$ and there is a lack of information about the impact of these changes on patient care.

The aim of this mixed-methods study is to determine the components of hospital service delivery of hip fracture care that predict patient outcomes post hip fracture, and the direct health costs attributable to these organisational factors. Furthermore, to understand factors that act as barriers and facilitators to the delivery of hip fracture care, including strategies that hospitals used to adapt and innovate hip fracture care delivery during the COVID-19 pandemic. Using these results, and working with key stakeholders, a toolkit will be developed, suitable for use by hospital managers, clinical leads and healthcare system leads across the country, to improve organisational delivery of high-quality hip fracture services. Understanding strategies hospitals used to reconfigure care during the COVID-19 pandemic will inform development of more resilient services in the future.

\section{METHODS AND ANALYSIS}

Quantitative study

Data sources: organisational level

Using a wide range of publicly available organisationallevel service data, available at a hospital/trust provider level in England and Wales, including eight national audits and nine data series/ratings resources (table 1), data will be extracted to characterise each component of the hip fracture care pathway from admission to discharge (figure 1). The derived organisational metrics will be linked using hospital provider codes. Time-specific organisational metrics will be linked to patients by using the year in which they were admitted with their hip fracture. Organisational metrics aim to quantify provision of emergency, orthopaedic, anaesthetic, orthogeriatric, nursing, physiotherapy, rehabilitation and governance services.

\section{Study population}

Data sources: patient-level

Using routinely collected Hospital Episodes Statistics (HES) Admitted Patient Care (APC) data, that includes admissions to all English hospitals within the NHS (ie, excluding privately financed healthcare), patients will be linked by NHS Digital, the national health and social care data provider, to Office for National Statistics (ONS) Civil Registrations (deaths) mortality data for the same period. Similarly, in Wales the NHS Wales Informatics Service will link patients with hip fractures in the Patient Episode Database for Wales (PEDW) to ONS mortality data. The resulting HES-ONS and PEDW-ONS patients' 
Table 1 Organisational datasets included in the REducing unwarranted variation in the Delivery of high qUality hip fraCture services in England and Wales study

\begin{tabular}{|c|c|c|c|c|}
\hline $\begin{array}{l}\text { Organisational level } \\
\text { dataset }\end{array}$ & $\begin{array}{l}\text { England } \\
\text { and Wales }\end{array}$ & Available years & Type of data available & Ref \\
\hline $\begin{array}{l}\text { NHFD Benchmark } \\
\text { Summary }\end{array}$ & Combined & 2016, 2017, 2018, 2019 & $\begin{array}{l}\text { Summary of hospital performance in three } \\
\text { areas: assessment, surgery, outcomes }\end{array}$ & 31 \\
\hline NHFD Best Practice & Combined & April 2016 to March 2019 & $\begin{array}{l}\text { The charts provide feedback on service } \\
\text { quality and compliance with national care } \\
\text { standards }\end{array}$ & 31 \\
\hline $\begin{array}{l}\text { NHFD Charts } \\
\text { (excluding BP and KPI) }\end{array}$ & Combined & April 2016 to March 2019 & $\begin{array}{l}\text { Charts with information on: } \\
\text { Anaesthesia. } \\
\text { Overall performance. } \\
\text { Patient safety. } \\
\text { Surgery used. }\end{array}$ & 31 \\
\hline
\end{tabular}

$\begin{array}{llll}\begin{array}{l}\text { Physiotherapy Hip } \\ \text { Fracture Sprint Audit }\end{array} & \text { Combined } & 2017 & \begin{array}{l}\text { Review of physiotherapy rehabilitation for } \\ \text { hip fracture patients in the UK }\end{array} \\ \begin{array}{l}\text { Fracture Liaison } \\ \text { Service Database }\end{array} & \text { Combined } & 2016,2017,2018,2019 & \begin{array}{l}\text { National audit of secondary fracture } \\ \text { prevention in England and Wales }\end{array} \\ \begin{array}{l}\text { Care Quality } \\ \text { Commission }\end{array} & \text { England } & 2016,2017,2018,2019 & \begin{array}{l}\text { CQC independent regulator of health and } \\ \text { adult social care in England }\end{array} \\ \begin{array}{l}\text { NHS Staff Survey } \\ \text { Themes }\end{array} & \text { England } & 2016,2017,2018,2019 & \begin{array}{l}\text { Reports how NHS staff in England } \\ \text { experience working for their respective NHS } \\ \text { organisations }\end{array}\end{array}$

\begin{tabular}{|c|c|c|c|c|}
\hline $\begin{array}{l}\text { NHS Workforce } \\
\text { Statistics }\end{array}$ & England & April 2016 to March 2019 & $\begin{array}{l}\text { Monthly numbers of NHS Hospital staff } \\
\text { groups working in Trusts in England as } \\
\text { headcount and full-time equivalents }\end{array}$ & 53 \\
\hline
\end{tabular}

NHS Bed Availability England April 2016 to March 2019

A quarterly collection from all NHS

and Occupancy Data-

Overnight

overnight

number of available bed days and the tota

number of occupied bed days by consultant main specialty.

\begin{tabular}{|c|c|c|c|c|}
\hline $\begin{array}{l}\text { NHS Supporting } \\
\text { Facilities Data } \\
\text { Operating Theatres }\end{array}$ & England & April 2016 to March 2019 & $\begin{array}{l}\text { The number of operating theatres and } \\
\text { supporting facilities in NHS organisations } \\
\text { (trusts) in England }\end{array}$ & 55 \\
\hline $\begin{array}{l}\text { NHS Emergency } \\
\text { Department } \\
\text { Attendances and } \\
\text { Emergency Admissions }\end{array}$ & England & April 2016 to March 2019 & $\begin{array}{l}\text { A\&E attendances and emergency admission } \\
\text { monthly statistics, NHS and independent } \\
\text { sector organisations in England }\end{array}$ & 56 \\
\hline NHS Staff & Wales & September 2016-2018, March 2019 & $\begin{array}{l}\text { Assignment count and full-time equivalent } \\
\text { of directly employed NHS staff by grade } \\
\text { and area of work }\end{array}$ & 57 \\
\hline $\begin{array}{l}\text { NHS Beds by } \\
\text { Specialty: Availability } \\
\text { and Occupancy Data }\end{array}$ & Wales & 2016-2017, 2017-2018, 2018-2019 & NHS Beds by organisation and specialty & 58 \\
\hline
\end{tabular}




\begin{tabular}{llll}
\hline Table 1 Continued & & & \\
\hline $\begin{array}{l}\text { Organisational level } \\
\text { dataset }\end{array}$ & $\begin{array}{l}\text { England } \\
\text { and Wales }\end{array}$ & Available years & Type of data available \\
\hline $\begin{array}{l}\text { NHS ED Attendances } \\
\text { and Emergency }\end{array}$ & Wales & April 2016 to March 2019 & $\begin{array}{l}\text { Reports performance against waiting } \\
\text { times targets by hospital. (Requested } \\
\text { Admissions }\end{array}$ \\
& & $\begin{array}{l}\text { and received from NWIS directly, total } \\
\text { emergency admissions.) }\end{array}$ \\
\hline
\end{tabular}

A\&E, accident and emergency; BP, Best Practice; CQC, Care Quality Commission; ED, Emergency Department; KPI, Key Performance Indicator; NHFD, National Hip Fracture Database; NHS, National Health Service; NWIS, NHS Wales Informatics Service.

data extracts will then be linked to data from the UK's National Hip Fracture Database (NHFD) for the admission. The NHFD, active since 2007 , is a clinically led webbased audit of hip fracture care and secondary fracture prevention in England, Wales and Northern Ireland, data collected have informed the Best Practice Tariff (BPT) for hip fracture care since $2010 .^{2021}$

HES/PEDW provide information on patient demographics, admission, discharge, clinical diagnoses using International Classification of Diseases Tenth Revision (ICD-10) disease codes, and Classification of Interventions and Procedures version 4 codes. $^{22}$ ONS mortality data are obtained from death certificates of all registered deaths in England and Wales, ${ }^{23}$ thus capturing deaths that occurred inside and outside of hospital. Each NHFD record includes information on patient demographics, anaesthetic risk grade, type of hip fracture and surgical operation performed.

Hip fracture admissions will be identified using ICD-10 codes for fractured neck of femur (S72.0), pertrochanteric fracture (S72.1), subtrochanteric fracture (S72.2) and unspecified fracture of femur (S72.9). The study

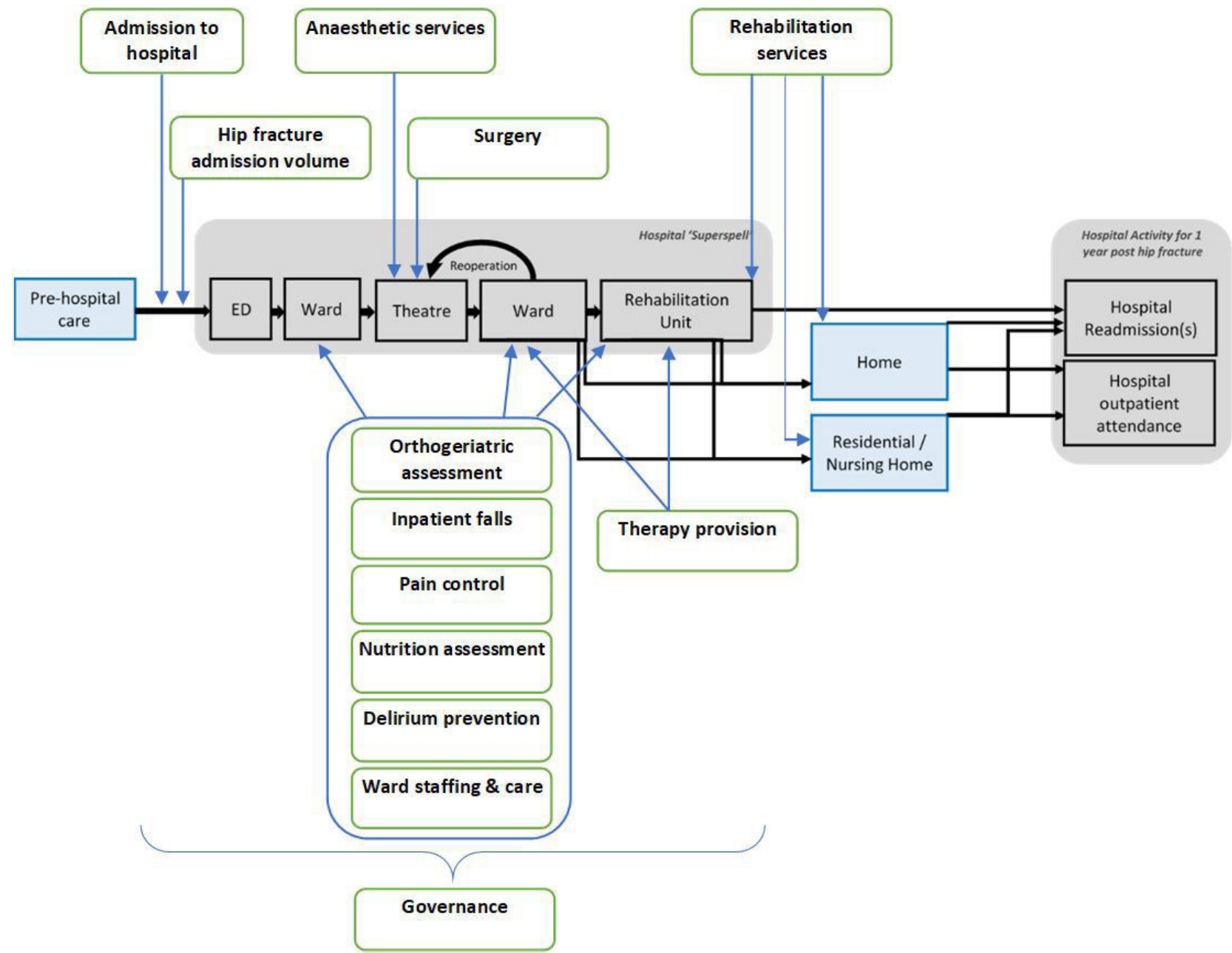

Figure 1 Hip fracture care pathway and domains of organisational-level data. Hip fracture care pathway flows from prehospital care, through the hospital superspell, through to any occurring hospital activity in the year after hip fracture. Organisational domains are indicated by green boxes, and these are mapped to the pathway. 
population will consist of index cases of hip fracture (ie, the first occurrence of hip fracture), among English or Welsh residents (male and female) aged 60 years or more, admitted to an English or Welsh hospital between 1 April 2016 and 31 March 2019.

\section{Patient-level outcomes}

For each patient with an index hip fracture, all HES APC, outpatient clinic and emergency department (ED) attendance data in England, and similar PEDW data in Wales, will be analysed for the subsequent 12 months enabling post-fracture follow-up (thus the last follow-up will complete 31 March 2020). Patient outcome measures will include: (i) cumulative mortality at 30 days and 1 year, (ii) acute NHS 'super-spell' (defined as the index hip fracture admission, plus if applicable, planned hospital transfers for elective care and/or subsequent unplanned hospital transfers for emergency care), (iii) return to original residence at discharge, (iv) emergency 30-day readmissions (defined as an emergency all-cause admission to any English/Welsh NHS hospital that occurred within 30 days of hospital discharge following a hip fracture superspell), (v) mobility at 120 days, (vi) return to original residence at 120 days, (vii) osteoporosis treatment to reduce future fracture risk, (viii) re-fracture/re-operation, (ix) the total number of days spent in hospital in the year following hip fracture, informing $(\mathrm{x})$ total direct health costs attributable to hip fracture (see below).

\section{Health cost outcomes}

HES data reports Healthcare Resource Groups assigned to each finished consultant episode in a hospital spell via the Casemix Grouper Software (HRG4+). ${ }^{24}$ HRGs are standard groups of clinically similar treatments that consume a common set of healthcare resources. HRGs will be valued using the most up-to-date prices available from Department of Health and Social care reference costs for NHS trusts, including a per diem costing for bed days in excess of those expected for a standard tariff. ${ }^{25}$

\section{Statistical analysis of outcomes}

Using a systematic approach, organisational factors will be identified which predict patient-level outcomes including associated health costs. Using a 'top-down' approach, defining a priori indicator groupings (domains), informed by NHFD BPT variables with stakeholder consensus, key indicators will be identified predicting patient outcomes. Clinicians will undertake expert panel review to select potential explanatory organisational-level variables. The flow diagram (figure 2) illustrates this review process which will be repeated across all organisational data sources.

Further expert-driven data reduction will involve examining the prevalence and correlation of selected organisational variables with each outcome, to finalise the dataset for multi-level models. Those variables selected for inclusion will be mapped to one or more domains of hip fracture care (eg, admission, anaesthesia, delirium

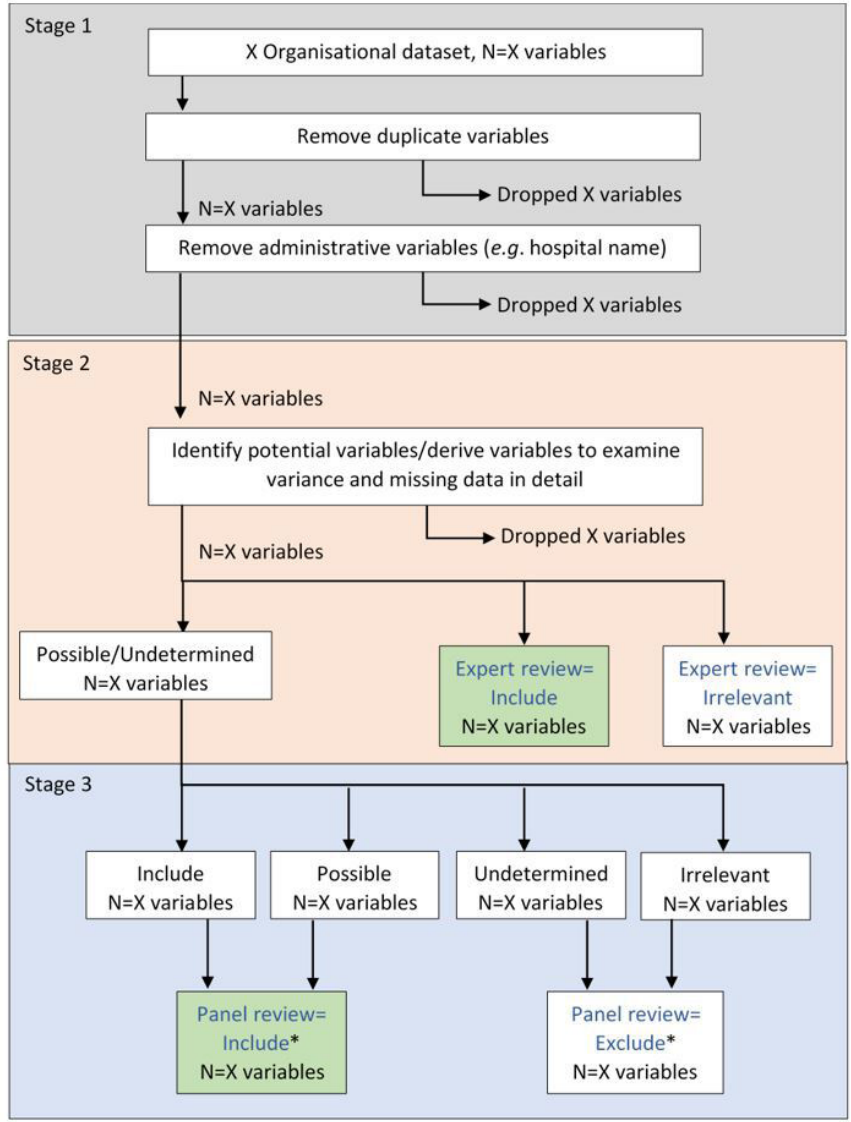

Figure 2 Flow diagram used to determine organisational data metrics. *Include if two or more of the four reviewers categorised variable as 'include' or 'possible', otherwise exclude; any lack of consensus resolved by a third reviewer (the principal investigator). Shaded boxes indicate variables which will be included in analyses.

prevention, governance, annual hospital admissions for hip fracture, inpatient falls, nutrition, orthogeriatrician assessment, pain management, rehabilitation, surgery, therapy provision and ward staffing and care (see figure 3). Each organisational variable will be assigned as relevant to one or more patient outcomes.

Multi-level regression modelling will identify organisational components of the hip fracture care pathway responsible for the greatest variation in patient outcomes and costs. The hierarchical data structure consists of patients (level 1), nested within hospitals (level 2). Multilevel regression models will describe the association of organisational-level factors on patient-level outcomes, while adjusting for patient case-mix, and allow assessment of interactions between patient-level and organisationallevel factors. Case-mix adjustment will be the same as that used in the NHFD clinical audit ${ }^{26}$ and will include age, sex, residence prior to admission, pre-fracture mobility, fracture type and American Society of Anaesthesiologists grade $^{27}$ (figure 3). Further adjustment will explore inclusion of the following additional patient-level variables as part of the case-mix adjustment: area-level deprivation, quantified by the Index of Multiple Deprivation ${ }^{28}$; 


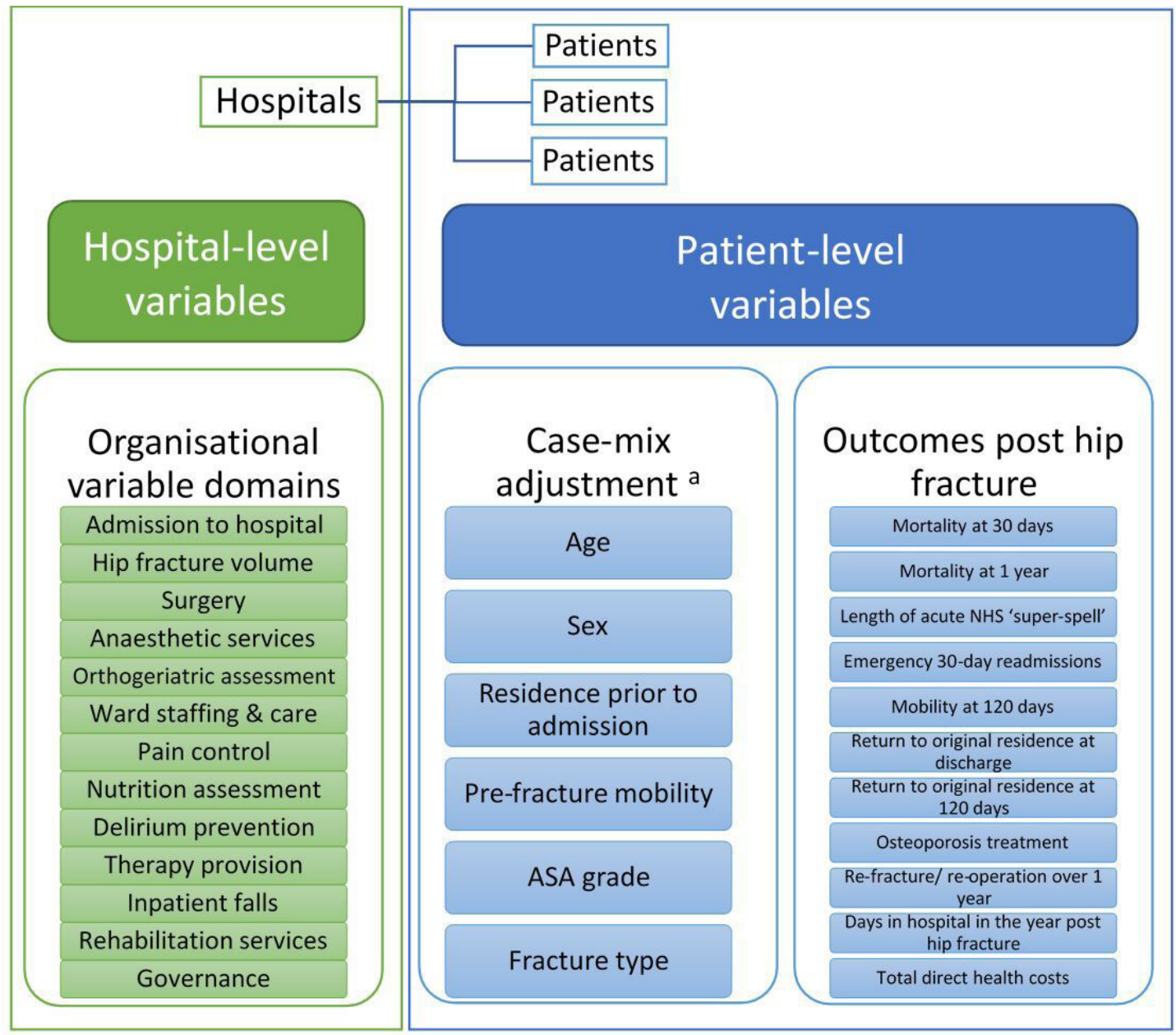

Figure 3 Structure of the multi-level models used in the statistical analyses in the REducing unwarranted variation in the Delivery of high qUality hip fraCture services in England and Wales study. ${ }^{2}$ Same as patient-level National Hip Fracture Database clinical audit case-mix variables. ${ }^{26}$ ASA, American Society of Anaesthesiologists.

comorbidity, quantified by the Charlson comorbidity index ${ }^{29}$ and frailty, quantified by the hospital frailty risk score. ${ }^{30}$

Having identified the proportion of variance in a given patient-level outcome, not explained by patient-level factors (ie, case-mix), the between-hospital variability explained by fixed organisational effects will be quantified, that is, the proportion of between-hospital variance explained by the configuration of specific services. For each outcome, domains added sequentially to a multilevel model (after case-mix adjustment) will be fitted to identify those domains which have the greatest effect and predict patient-level outcomes. From the selected domains the most influential organisational variables will be identified.

\section{Statistical analysis of costs}

Patient costs will be adjusted for baseline costs (ie, the healthcare costs incurred in the year prior to hip fracture) and patient case-mix as detailed earlier. Adjusted estimates of patient costs will be reported as aggregate costs and costs disaggregated by initial hospital superspell and readmissions/further NHS care. Different pathways of care following hip fracture will be identified by expert consensus agreement for comparative cost analyses.

The multi-level model will be used to determine how health costs relate to organisational factors and patient outcomes, identifying organisational factors associated with highest/lowest costs. Cost profiles will be calculated for different hip fracture care pathway models, facilitating budget impact analyses (eg, estimate potential NHS savings should hip fracture care pathway models change). Costed scenarios will inform the Implementation Toolkit cost-benefit calculator.

\section{Qualitative study}

Service delivery stakeholder interviews

For the qualitative interviews four hospitals will be selected and approached. For English hospitals, BPT (2017-2019) and Key Performance Indicators (2018) will be collated, and 25 hospitals with the most and 25 with the least variable scores selected. ${ }^{31}$ Hospitals will be excluded if (i) they are major trauma centres (as these hospitals may differ significantly from the majority of hip fracture hospitals which are not major trauma centres); (ii) they have recently merged or moved location; (iii) 
they are participating in the hip fracture quality improvement programme (as practices in these hospitals are likely to be changing); (iv) they are located local to the study team (eg, Bristol, Bath) (to avert any conflict of interest within the research team); (v) if data quality is poor (as measured by NHFD report ${ }^{1}$ ) ; (vi) or if the hospital manages low volumes of hip fractures (annual hip fracture admissions less than the fifth percentile). From the remaining pool, hospitals will be selected to provide a diverse range in terms of 30-day mortality trend (2017-2018), BPT trend (2017-2019), hospital size (large/medium) and geographical location (eg, city/ rural/coastal, north/south of England).

A qualitative interview study will characterise the organisation of hip fracture services and identify barriers and facilitators to the implementation of key components of fracture care. In addition, strategies that hospitals used to adapt and innovate hip fracture care delivery, during the COVID-19 pandemic, along with barriers and facilitators to the reorganisation of services in 2020 will be ascertained. Four hospitals have been identified that encompass variation in a range of characteristics as listed above, aiming to identify varied service configurations. ${ }^{32}$ Studying care in these settings will enable us to capture the experiences of those delivering different models of hospital care.

At the four participating hospitals, 1:1 in-depth interviews will be conducted with stakeholders involved in the organisation and delivery of hip fracture services, including orthogeriatricians, orthopaedic surgeons, anaesthetists, emergency medicine physicians, physiotherapists, occupational therapists, trauma nurses and discharge and service managers. Interviews will be carried out either remotely or in person (according to infection control constraints) with informed consent, including consent to audio-recording. An estimated 10-15 professionals will be interviewed at each hospital site, totalling around 40-60 professionals across the study. However, final sample size will be determined when data saturation is achieved; that is, when no new themes or subthemes are identified in the data. ${ }^{33}$ Interviews will be conducted using a topic guide with a list of themes and subthemes to guide discussions. This will enable us to compare and contrast stakeholder views and provide flexibility to pursue emerging ideas. ${ }^{34}$ To understand contextual factors that impact on service implementation, study design and analysis will be informed by Implementation Science. Implementation Science comprises theories or frameworks that have been used to help understand factors that help or hinder the delivery of complex interventions such as hip fracture care ${ }^{35-38}$ The topic guide has been devised by the study team with Patient and Public Involvement (PPI). PPI identified patient priorities during the first wave of COVID-19, which will enable exploration of how services adapted to meet these needs. Four to six pilot interviews will inform topic guide refinement. If refinements are minor, initial pilot interviews will contribute to the main analysis.
Interviews will be audio-recorded, transcribed and anonymised. Transcripts will be imported into NVivo qualitative software and analysed using an inductive thematic analysis to identify key themes and subthemes in the data. ${ }^{39}$ Following this, an abductive approach will be used whereby themes/subthemes will be transposed onto concepts from Implementation Science theory. ${ }^{40}$ To ensure rigour, $20 \%$ of transcripts will be independently analysed in duplicate and themes reviewed and refined to agree a themes list.

\section{Documentary analysis of BOA reports}

To complement the qualitative interviews and to understand the common themes and solutions ('lessons learnt') in relation to provision of hip fracture care, qualitative content analysis will be conducted of anonymised detailed hospital-initiated peer-review process (PRP) reports from 22 hospitals (all that have been conducted by the BOA to 2019). PRP reports, produced by the BOA over the period 2013-2019, were delivered when UK hospital service leads requested a BOA 'peer-review' to improve their hip fracture service. Each PRP report includes interviews with a range of staff (eg, clinical directors, clinicians, nurses, therapists, anaesthetists, ED personnel and managers). PRP reports are structured encompassing appraisal of the full hip fracture care pathway: ED, orthopaedics, anaesthetics, theatre activity, orthogeriatrics, nursing, therapies, discharge planning, collection of NHFD audit data and governance structures. Reports list all areas of good practice and highlight issues where improvements are achievable. PRP reports include a series of recommendations made by the multi-disciplinary assessment team with numerated action points. Reports will be imported into NVivo qualitative analysis software and will be analysed thematically to identify barriers and facilitators to implementation of quality hip fracture services. ${ }^{39}$ Themes identified in the documentary analysis will be mapped onto those from the qualitative interviews. To illustrate this process, data will be displayed on charts using the framework approach to data organisation. ${ }^{41}$ Written accounts will then be generated. Recommendations will inform choice of domains in quantitative analyses and of subsequent Implementation Toolkit development.

\section{Implementation Toolkit development}

In 2015 the Royal (formerly National) Osteoporosis Society (ROS), developed a Fracture Liaison Service (FLS) Toolkit to aid FLS commissioning of new or improved services. ${ }^{42}$ It was designed to support business case development, saving time for service leads and commissioners, and has been highly successful. Since 2015 the Service Improvement Team at the ROS have supported the development of 34 new FLSs. These new services cover a patient population of more than 12 million people and it has been estimated that 5 years following implementation of all these services, approximately 3854 hip fractures will have been prevented. Approximately $60 \%$ of the UK population can access an FLS, and the ROS is currently 
working with sites all over the UK to ensure that current services are delivering in line with national clinical guidelines, and supporting sites that do not have an FLS at present. The ROS has acquired extensive experience in service improvement, refining tools based on user feedback, and continuing to support quality improvement.

Working with the ROS, the BOA and other stakeholders, a Hip Fracture Implementation Toolkit will be codeveloped, focusing on inpatient hip fracture services, prioritising organisational factors identified from our quantitative and qualitative results which contribute to poor and/or highly variable patient outcomes post hip fracture. All acute NHS hospitals currently have a hip fracture care pathway; hence, the toolkit will include a step-by-step guide to improve and implement changes to current services. It will be made available online and provide a series of instructions and guides (ie, 'tools') for managers, clinical leads and healthcare system leads to use to improve their hospital hip fracture services, encompassing service redesign/restructuring, organisational cultural change, and approaches to improve efficient use of limited healthcare resources. Tools will include a service improvement guide, business case resources, a cost calculator and a project plan.

\section{Patient and public involvement}

This study has been developed in collaboration with the University of Bristol Musculoskeletal Research Unit (MRU) PPI group comprising members who have had/ are having treatment for osteoporosis and/or fractures, who meet (currently virtually) every 3 months to input into the design and conduct of MRU research projects. They have guided development of the project proposal, informing prioritisation of research questions, drafting plain English text, changing language and wording within study texts; they have informed methods adaptation in response to COVID-19 in 2020. The group will provide ongoing support throughout the study, addressing (i) key patient and carer questions and priorities, (ii) the interpretation and relevance of results and (iii) communication of findings. 'Taking the research' to patients in the involvement group rather than asking patients to attend research management meetings has improved engagement and fosters strong collaboration and respect. ${ }^{43}$ Meetings will be organised by an experienced PPI coordinator (KW), who will facilitate meetings, provide ongoing support and tailored development to patient members, and advise on good practice.

\section{ETHICS AND DISSEMINATION Ethics and governance}

The quantitative study has research approvals from NHS Health Research Authority-London City and East Research Ethics Committee (20/LO/0101, 11/02/2020); Royal College of Physicians (RCP) Falls and Fragility Fracture Audit Programme (FFFAP) (FFFAP/2018/003, $11 / 12 / 2019)$ and Healthcare Quality Improvement
Partnership approval (HQIP330, 18/06/2020); NHS Wales Informatics Service (NWIS) (30941, 13/03/2020) and an NHS Digital Data Sharing Agreement (DARS-NIC334549-B1Y6X-v1.4, 28/09/2020). The qualitative study has been approved for conduct by the Faculty of Health Sciences University of Bristol Research Ethics Committee (Ref: 108284, 9/9/2020) and by the NHS Health Research Authority (20/HRA/71, 10/9/2020). Each NHS Trust has provided Research and Development approval.

\section{Dissemination}

Findings will be disseminated through scientific conferences, peer-reviewed publications and online implementation workshops. Results will be fed back to the scientific committee with oversight of the NHFD as well as the BOA. Reports will be provided to each hospital in England and Wales summarising findings, and the PPI group will be involved in all stages of dissemination. Working with the ROS, dissemination materials will be developed for its membership network $(\mathrm{n}=20000)$. The Implementation Toolkit will be hosted and made freely available by the ROS website.

\section{CONCLUSION}

Through the use of mixed methodology, this study will determine the components of hospital service delivery which account for poor patient outcomes post hip fracture and identify which service configurations are most efficient and successful. Quantitative analyses will allow us to distinguish patient outcomes explained by the health of the patient themselves, versus outcomes attributable to the hospital services which they encounter. Domains of hip fracture care which are most critical for a wide range of patient outcomes over 12 months, will be identified. The direct health costs ascribed to each patient in the year after hip fracture, accounting for costs in the year prior to fracture, will be calculated. Thus, hospital expenditure attributable to different components of hip fracture service delivery will be calculated. These financial calculations will inform cost calculators in the Implementation Toolkit. The Toolkit will be a novel, freely available online resource for managers, clinical leads and healthcare system leads to use to improve their hip fracture service.

The qualitative analysis will aid understanding of the organisational processes that help or hinder the implementation of key components of hip fracture services. In addition, it will identify strategies that hospitals have used to adapt and innovate hip fracture care delivery during the COVID-19 pandemic. Knowledge gained will inform toolkit development, aiming to assist services in overcoming organisational barriers when designing and implementing sustainable high-quality fracture services, and improve patient care. Understanding strategies hospitals used to reconfigure care during the COVID-19 pandemic will provide learning towards the development of more robust and resilient hip fracture services in the future. This information is likely to be transferrable to other services. 
In conclusion, a system-wide understanding of sources of variation in hip fracture care delivery and the effects on patient outcomes will inform service-level interventions to reduce unwarranted variation, maximise health equity and ultimately improve patient experience. The study aims to show effective hip fracture care is more efficient, realising cost-savings in hospital bed-days potentially re-directable to other services. This project is novel in terms of its scale and the unique datasets which gives us a rare opportunity to robustly assess what is a complex system of care and the very real impacts this system has on patients. Findings will inform future commissioning/ service planning priorities for hip fracture care, inform national review processes for hip fracture services and, together with a new online toolkit, this programme aims to minimise avoidable variation in hip fracture care and improve the quality of care for patients across the UK.

\section{Author affiliations}

${ }^{1}$ Musculoskeletal Research Unit, Translational Health Sciences, Bristol Medical School, University of Bristol, Bristol, UK

${ }^{2}$ Division of Population Medicine, School of Medicine, Cardiff University and University Hospital of Wales, Cardiff, UK

${ }^{3}$ National Hip Fracture Database, Royal College of Physicians, London, UK ${ }^{4}$ Department of Trauma and Orthopaedics, Southmead Hospital, North Bristol NHS Trust, Bristol, UK

${ }^{5}$ Nuffield Department of Orthopaedics, Rheumatology and Musculoskeletal Sciences, University of Oxford, Oxford, UK

${ }^{6}$ Division of Orthopaedics, Barts and The London School of Medicine and Dentistry, Queen Mary University of London, London, UK

${ }^{7}$ Royal London Hospital, Barts Health NHS Trust, London, UK

${ }^{8}$ Clinical \& Operations Directorate, Royal Osteoporosis Society, Bath, UK

${ }^{9} \mathrm{NIHR}$ Biomedical Research Centre at University Hospitals Bristol and Weston NHS Foundation Trust and the University of Bristol, Bristol, UK

${ }^{10}$ Population Health Sciences, Bristol Medical School, University of Bristol, Bristol, UK

Twitter Xavier L Griffin @xlgriffin, Katie Whale @whalekatie and Celia L Gregson @ celiagregson

Contributors CLG, RP, SD, AJohansen, TC, MKJ, XLG, TJ, JG, KW, ECB, EMRM, YB-S, RG-H and AJudge are responsible for the study design. CLG, AJudge, RG-H, $\mathrm{RP}, \mathrm{SD}$ and $\mathrm{MB}$ are responsible for study management and coordination. RP, SD and CLG drafted the paper. All authors read, commented on and approved the final manuscript.

Funding This work is funded by Versus Arthritis (reference 22086). This study is supported by the NIHR Biomedical Research Centre at University Hospitals Bristol and Weston NHS Foundation Trust and the University of Bristol. MKJ is supported by the National Institute for Health Research (NIHR) Oxford Biomedical Research Centre (BRC). The views expressed are those of the author(s) and not necessarily those of the NHS, NIHR or the Department of Health and Social Care.

Competing interests None for RP, SD, XLG, TJ, JG, MB, KW, ECB, YB-S, RG-H, CLG. AJohansen is the clinical lead for the National Hip Fracture Database, in the Royal College of Physicians, London. TC is the British Orthopaedic Association (BOA) representative and sits on the board of the Falls and Fragility Fracture Audit Project (which includes the National Hip Fracture Database), he helped set up and perform many of the BOA multidisciplinary peer reviews for hip fractures, he has design and educational contracts with Stryker, Acumed and Swemac. MKJ has received honoraria, unrestricted research grants, travel and/or subsistence expenses from: Amgen, Consilient Health, Kyowa Kirin Hakin, UCB, Abbvie. EMRM has received funds for an unrelated independent study sponsored by CeramTec UK limited. AJudge has received consultancy fees from Freshfields, Bruckhaus, Derringer and Anthera Pharmaceuticals Ltd.

Patient and public involvement Patients and/or the public were involved in the design, or conduct, or reporting, or dissemination plans of this research. Refer to the Methods section for further details.
Patient consent for publication Not required.

Provenance and peer review Not commissioned; externally peer reviewed.

Open access This is an open access article distributed in accordance with the Creative Commons Attribution 4.0 Unported (CC BY 4.0) license, which permits others to copy, redistribute, remix, transform and build upon this work for any purpose, provided the original work is properly cited, a link to the licence is given, and indication of whether changes were made. See: https://creativecommons.org/ licenses/by/4.0/.

\section{ORCID iDs}

Rita Patel http://orcid.org/0000-0002-9136-9529

Sarah Drew https://orcid.org/0000-0002-2092-8506

Tim Chesser http://orcid.org/0000-0001-6837-6029

Muhammad K Javaid http://orcid.org/0000-0001-7985-0048

Xavier L Griffin http://orcid.org/0000-0003-2976-7523

Katie Whale http://orcid.org/0000-0002-0012-7103

Estela Capelas Barbosa http://orcid.org/0000-0002-7621-7957

Elsa M R Marques http://orcid.org/0000-0003-1360-5677

Yoav Ben-Shlomo http://orcid.org/0000-0001-6648-3007

Rachael Gooberman-Hill http://orcid.org/0000-0003-3353-2882

Andrew Judge http://orcid.org/0000-0003-3015-0432

Celia L Gregson http://orcid.org/0000-0001-6414-0529

\section{REFERENCES}

1 Royal College of Physicians. National hip fracture database (NHFD) annual report 2019. London: RCP, 2019.

2 Griffin XL, Parsons N, Achten J, et al. Recovery of health-related quality of life in a United Kingdom hip fracture population. The Warwick Hip Trauma Evaluation--a prospective cohort study. Bone Joint J 2015;97-B:372-82.

3 Hawley S, Javaid MK, Prieto-Alhambra D, et al. Clinical effectiveness of orthogeriatric and fracture liaison service models of care for hip fracture patients: population-based longitudinal study. Age Ageing 2016;45:236-42.

4 Glynn J, Hollingworth W, Bhimjiyani A, et al. How does deprivation influence secondary care costs after hip fracture? Osteoporos Int 2020;31:1573-85.

5 National Institute for Health and Care Excellence (NICE). Hip fracture in adults: Quality standard [QS16]. Manchester: NICE, 2017.

6 National Institute for Health and Care Excellence. Hip fracture: the management of hip fracture in adults. NICE clinical guideline 124. Manchester: NICE, 2011.

7 Scottish Intercollegiate Network (SIGN). Sign guidelines 111 management of hip fracture in older people, 2010. Available: http:// signacuk/guidelines/fulltext/111/indexhtml

8 Royal College of Physicians. National hip fracture database (NHFD) annual report 2017. London: RCP, 2017.

9 Royal College of Physicians. National hip fracture database (NHFD) annual report 2016. London: RCP, 2016.

10 Royal College of Physicians. National hip fracture database (NHFD) extended report. RCP London, 2014.

11 Patel R, Bhimjiyani A, Ben-Shlomo Y, et al. Social deprivation predicts adverse health outcomes after hospital admission with hip fracture in England. Osteoporos Int 2021;5:1-3.

12 The NHS Information Centre. Emergency readmissions 201011 summary, 2013. Available: https://www.gov.uk/government/ publications/emergency-readmissions-data

13 lacobucci G. Covid-19: all non-urgent elective surgery is suspended for at least three months in England. BMJ 2020;368:m1106.

14 Royal College of Surgeons. Clinical guide to surgical prioritisation during the coronavirus pandemic, 2020.

15 NHS England. Clinical guide for the management of trauma and orthopaedic patients during the coronavirus pandemic, 2020.

16 NHS England. Clinical guide for the perioperative care of people with fragility fractures during the coronavirus pandemic, 2020.

17 British Orthopaedic Association. Emergency BOAST: management of patients with urgent orthopaedic conditions and trauma during the coronavirus pandemic, 2020.

18 Williams JT, Kumar A. The effect of the COVID-19 pandemic on hip fracture care. British Orthopaedic Association, 2020.

19 Cronin M, Mullins M, Pathmanaban P, et al. COVID-19 causes a SHiFT in the sands for proximal femoral fracture management? British Orthopaedic Association, 2020.

20 National hip fracture database (NHFD). Available: https://www. rcplondon.ac.uk/projects/national-hip-fracture-database-nhfd [Accessed 18 Nov 2020]. 
21 NHS England and NHS Improvement website. Available: https:// improvement.nhs.uk/documents/484/Annex_DtD_Best_practice_ tariffs.pdf [Accessed 18 Nov 2020].

22 Health and Social Care Information Centre. HES data dictionary: admitted patient care, 2017

23 NHS Digital. Linked HES-ONS mortality data [Website], 2018. Available: https://digital.nhs.uk/data-and-information/data-tools-andservices/data-services/linked-hes-ons-mortality-data [Accessed 25 May 2018].

24 Health \& Social Care Information Centre. Casemix grouper, 2013. Available: http://www.hscic.gov.uk/casemix-grouper

25 Department of Health. NHS reference costs 2012 to 2013, 2013. Available: https://www.gov.uk/government/publications/nhsreference-costs-2012-to-2013

26 NHFD 2019 annual report: methods used for statistical analysis. Available: https://www.nhfd.co.uk/files/2019ReportFiles/NHFD_ Statistical_Methods_Update_2019.pdf

27 American Society of Aneshthesiology: new classification of physical status. Anesthesiology: The Journal of the American Society of Anesthesiologists 1963;24:111.

28 Department for Communities and Local Government. The English Indices of Deprivation 2010: technical report, 2011.

29 Armitage JN, van der Meulen JH, Royal College of Surgeons Comorbidity Consensus Group. Identifying co-morbidity in surgical patients using administrative data with the Royal College of surgeons Charlson score. Br J Surg 2010;97:772-81.

30 Gilbert T, Neuburger J, Kraindler J, et al. Development and validation of a hospital frailty risk score focusing on older people in acute care settings using electronic hospital records: an observational study. Lancet 2018;391:1775-82.

31 Royal College of Physicians. National hip fracture database: charts and reports. Available: https://www.nhfd.co.uk/20/NHFDCharts.nsf

32 Coyne IT. Sampling in qualitative research. purposeful and theoretical sampling; merging or clear boundaries? J Adv Nurs 1997;26:623-30.

33 Saumure K, Given LM. Data saturation. In: LM G, ed. The SAGE encyclopedia of qualitative research methods. Thousand Oaks, CA: SAGE Publications, Inc, 2008: 196-7.

34 Qualitative researching with text, image and sound. London 2000.

35 Peters DH, Adam T, Alonge O, et al. Implementation research: what it is and how to do it. BMJ 2013;347:f6753.

36 Greenhalgh T, Wherton J, Papoutsi C, et al. Beyond adoption: a new framework for theorizing and evaluating Nonadoption, abandonment, and challenges to the scale-up, spread, and sustainability of health and care technologies. J Med Internet Res 2017;19:e367.

37 Eccles MP, Mittman BS. Welcome to implementation scienceSpringer, 2006.

38 SAGE Publications Inc. The SAGE encyclopedia of qualitative research methods. Thousand Oaks, CA, 2008.

39 Braun V, Clarke V. Using thematic analysis in psychology. Qual Res Psychol 2006;3:77-101.

40 Timmermans S, Tavory I. Theory construction in qualitative research: from Grounded theory to Abductive analysis. Sociological Theory 2012;30:167-86.
41 Ritchie J, Lewis J, Nicholls CM, et al. Qualitative research practice : a guide for social science students and researchers. 2nd edn. Los Angeles: SAGE, 2014: 430.

42 National Osteoporosis Society. FLS implementation toolkit. Available: https://nos.org.uk/for-health-professionals/service-development/ fracture-liaison-services/fls-implementation-toolkit/

43 Gooberman-Hill R, Burston A, Clark E, et al. Involving patients in research: considering good practice. Musculoskeletal Care 2013;11:187-90.

44 Royal College of Physicians. National hip fracture database (NHFD) annual report 2018. London: RCP, 2018.

45 Royal College of Physicians. National audit of inpatient falls; audit report. London: RCP, 2015.

46 Royal College of Physicians. National audit of inpatient falls; audit report. London: RCP, 2017.

47 Royal College of Physicians. Recovering after a hip fracture: helping people understand physiotherapy in the NHS. Physiotherapy 'hip sprint' audit report 2017. London: RCP, 2018.

48 Royal College of Physicians. FLS forward: identifying high-quality care in the NHS for secondary fracture prevention. London: RCP, 2017.

49 Royal College of Physicians. Fracture liaison service database (FLS-DB) facilities audit. FLS breakpoint: opportunities for improving patient care following a fragility fracture. London: RCP, 2016.

50 Royal College of Physicians. Fracture Liaison Service Database (FLS DB) annual report. Leading FLS improvement: secondary fracture prevention in the NHS.London: RCP, 2017.

51 Care Quality Commission (CQC). Available: https://www.cqc.org.uk/ about-us/transparency/using-cqc-data

52 National NHS staff survey co-ordination centre. NHS staff survey. Available: https://www.nhsstaffsurveys.com/Page/1056/Home/NHSStaff-Survey-2020/

53 NHS workforce statistics. Available: http://content.digital.nhs.uk/ catalogue/PUB16973

54 NHS England. NHS bed availability and occupancy. Available: https://www.england.nhs.uk/statistics/statistical-work-areas/bedavailability-and-occupancy/

55 NHS England. Supporting facilities data. Available: https://www. england.nhs.uk/statistics/statistical-work-areas/cancelled-electiveoperations/supporting-facilities-data/

56 NHS England data. Available: https://www.england.nhs.uk/statistics/ statistical-work-areas/

57 StatsWales. NHS staff. Available: https://statswales.gov.wales/ Catalogue/Health-and-Social-Care/NHS-Staff/Medical-and-DentalStaff/hospitalmedicalanddentalstaff-by-specialty-year

58 StatsWales. NHS beds by specialty. Available: https://statswales. gov.wales/Catalogue/Health-and-Social-Care/NHS-Hospital-Activity/ NHS-Beds/nhsbeds-by-specialty

59 StatsWales. Accident and emergency. Available: https://statswales. gov.wales/Catalogue/Health-and-Social-Care/NHS-Hospital-WaitingTimes/Accident-and-Emergency 